\title{
Detection Of Malarial Parasite Using Microscopic RBC Images
}

\author{
${ }^{1}$ Sindhu M, ${ }^{2}$ Vivekanandan S J, ${ }^{3}$ Dr Sivasubramanian S \\ ${ }^{1}$ M.E student, Department of Computer Science and Engineering, Dhanalakshmi \\ College of Engineering, Chennai, Tamil Nadu, India
}
${ }^{2}$ Assistant Professor, Department of Computer Science and Engineering, Dhanalakshmi College of Engineering , Chennai, Tamil Nadu, India

\author{
${ }^{3}$ Associate Professor, Department of Computer Science and Engineering, \\ Dhanalakshmi College of Engineering , Chennai, Tamil Nadu, India
}

Iyessindhu.sss@gmail.com, ${ }^{2}$ vivekanandan.sj@dce.edu.in, ${ }^{3}$ sivasubramanian.s@dce.edu.in

\begin{abstract}
Malaria is an infectious sickness that influences a large number of lives each year. Customary conclusion of malaria in lab requires an accomplished individual and cautious investigation to segregate sound and contaminated red platelets (RBCs). It is likewise exceptionally tedious and may deliver wrong reports because of human mistakes. The target of this paper is to show how profound learning engineering, for example, convolutional neural organization (CNN) and Resnet-50 which can be valuable continuously malaria identification successfully and precisely from input pictures and to lessen difficult work with a portable application. To this end, we assess the presentation of a custom CNN model utilizing a repetitive stochastic inclination plummet (SGD) enhancer with a programmed learning rate locater and get an exactness in characterizing sound and tainted cell pictures with a serious level of accuracy and affectability. This result of the paper will work with microscopy determination of malaria to a portable application so unwavering quality of the therapy and absence of clinical mastery can be settled.
\end{abstract}

Keywords: Resnet-50, CNN, SGD, RBC.

\section{INTRODUCTION}

Psychological figuring recreates the manner in which people tackle issues while man-made reasoning and AI strategies look for making novel ways for taking care of issues that people can possibly improve. A generous measure of exploration has been finished during the last many years utilizing AI calculations for savvy answers for help medical care experts in lessening illnesses. Intestinal sickness illness began from Plasmodium parasites through mosquito-borne disease. Intestinal sickness is exceptionally normal over the world mostly in tropical locales. Intestinal sickness is generally spread across the globe. At the point when tainted female Anopheles mosquitoes nibble an individual, the parasites go into the blood and start harming red platelets (RBC) that convey oxygen. Seasonal infection is the intestinal sickness' first indication. The indication by and large beginnings in barely any days or weeks. Above all, the deadly parasites can remain alive over a year in an individual's body without showing any manifestations. In this way, a late treatment can cause difficulties and even passing. Consequently, numerous lives can be saved through early intestinal sickness discovery. Practically half of the populace on the planet is in peril from intestinal sickness. There are in excess of 200 million malaria cases and 400,000 passings answered each year because of intestinal sickness. Practically speaking, to distinguish intestinal sickness, microscopists assess blood (good and bad) spreads for illness analysis and ascertain parasitemia. Microscopy assessment is utilized as one of the excellent norms for the finding of intestinal sickness [1,2] to recognize the presence of parasites in a blood drop from thick blood spreads. Be that as it may, slight blood spreads are utilized for recognizing the types of parasite and the advancement of malaria stages. Assessment through a magnifying lens is generally utilized since it is modest yet tedious. The assessment precision depends on the nature of blood smear and a gifted individual who is master in the arrangement and assessment of 
uninfected and parasitized platelets.

\section{CNN}

A convolutional neural organization (CNN) is a sort of fake neural organization utilized in picture acknowledgment and handling that is explicitly intended to deal with pixel dataThere are three kinds of layers that make up the CNN which are the convolutional layers, pooling layers, and completely associated (FC) layers. At the point when these layers are stacked, a CNN engineering will be shaped. Little reliance on pre handling, diminishing the requirements of human exertion fostering its functionalities. It is straightforward and quick to execute. It has the most noteworthy precision among all alghoritms that predicts pictures. the more convolutional layers the better (sensibly speaking, as each convolutional layer lessens the quantity of information highlights to the completely associated layers), albeit after around a few layers the exactness acquire turns out to be somewhat little so you need to choose whether ... Tuning can begin! The hunt work takes as information the preparation information and an approval split to perform hyperparameter mixes assessment. The ages boundary is utilized in arbitrary inquiry and Bayesian Optimization to characterize the quantity of preparing ages for each hyperparameter mix

\section{ResNet-50}

ResNet-50 is a convolutional neural organization that is 50 layers profound. You can stack a pretrained adaptation of the organization prepared on in excess of 1,000,000 pictures from the ImageNet information base. The ResNet-50 model comprises of 5 phases each with a convolution and Identity block. Every convolution block has 3 convolution layers and every character block additionally has 3 convolution layers. The ResNet-50 has more than 23 million teachable boundaries. A lingering neural organization (ResNet) is a fake neural organization (ANN) of a sort that expands on builds known from pyramidal cells in the cerebral cortex. Remaining neural organizations do this by using skip associations, or alternate ways to bounce over certain layers. Perhaps the greatest benefit of the ResNet is while expanding network profundity, it keeps away from adverse results. So we can build the profundity however we have quick preparing and higher exactness.

\section{MeThODOLOGY}

This methodology is applied to achieve the objectives of the study. This method is about CNN algorithm that is based on neural network approach. In this paper the algorithm improves the performance . In this section the proposed methodology is given below in the detail.

\section{Module Description}

$>$ Pre-processing

$>$ Segmentation

$>$ Feature Extraction

$>$ Classification

\section{Pre-processing}

Preprocessing is for the most part applied to work on the nature of the picture and to lessen varieties in the pictures that would superfluously entangle the ensuing handling steps. Three key goals can be recognized: commotion expulsion, contrast improvement, brightening and staining adjustment.

For commotion evacuation, the most mainstream approaches have been grounded channels, like mean and middle channels, or Gaussian low-pass separating. Also, applying morphologic tasks is extremely mainstream. For contrast improvement, contrast extending procedures and histogram adjustment specifically, have been the most mainstream draws near. For light and staining varieties, shading standardization methods have been applied, including the mainstream utilization of grayscale colors.

There are various Pre-handling methods utilized in picture preparing for the improvement of the pictures. Dark scale transformation, resizing of the picture, increment the brilliance of the pictures and other pre-preparing methods are applied to change the picture into wanted arrangement for the following fragment . Pre-characterized and in-fabricated sifting strategies are applied for the better 
difference on a picture and furthermore different methods for numerous measurement for pictures are applied . The fundamental target of pre-preparing are

Resizing the image.

Reduce or eliminate noise

Enhancing the image contrast for visual evaluation

\section{Segmentation}

The interaction is utilized to separate picture into items and area. Addressing a picture into a lot less complex and more reasonable, which can be not difficult to break down in the following interaction division is utilized. This assists with investigating the code more straightforward. It fragments the picture pixels dependent on locale of homogeneity by separating certain highlights which are in like manner. It likewise eliminates the commotion present in the pictures. A definitive objective is to separate between the platelets and foundation. The picture acquired by division as displayed in figure 3 are given to the following cycle. The cells that have been recognized as perhaps tainted are then extricated from the picture and passed to the following phase of the calculation for highlight extraction.

By far most of these procedures are thresholding methods, for example, Otsu thresholding in mix with morphologic activities. Be that as it may, these strategies may not be ruling a result of their prevalent exhibition contrasted and different techniques, but instead in view of their relative straightforwardness. Different strategies incorporate Hough change, which makes presumptions about the platelet shape, and unaided k-implies pixel grouping. Cell division should be precise to figure the right parasitemia. Notwithstanding, contacting cells specifically confound the ID and division of individual cells. For this issue, strategies like watershed and dynamic shapes have been applied

The division circumstance for thick spreads is diverse in that white platelets and parasites should be portioned. In any case, white platelets are greater than red platelets and have more surface, which makes their division a lot simpler. Besides, white platelets simply should be distinguished and not to be handled or grouped further. Furthermore, parasites are exceptionally little and their dependable ID is generally significant. Thusly, the location of these items is for all intents and purposes more significant than their division, which may clarify again the predominance of thresholding strategies and morphologic tasks.

Figure 

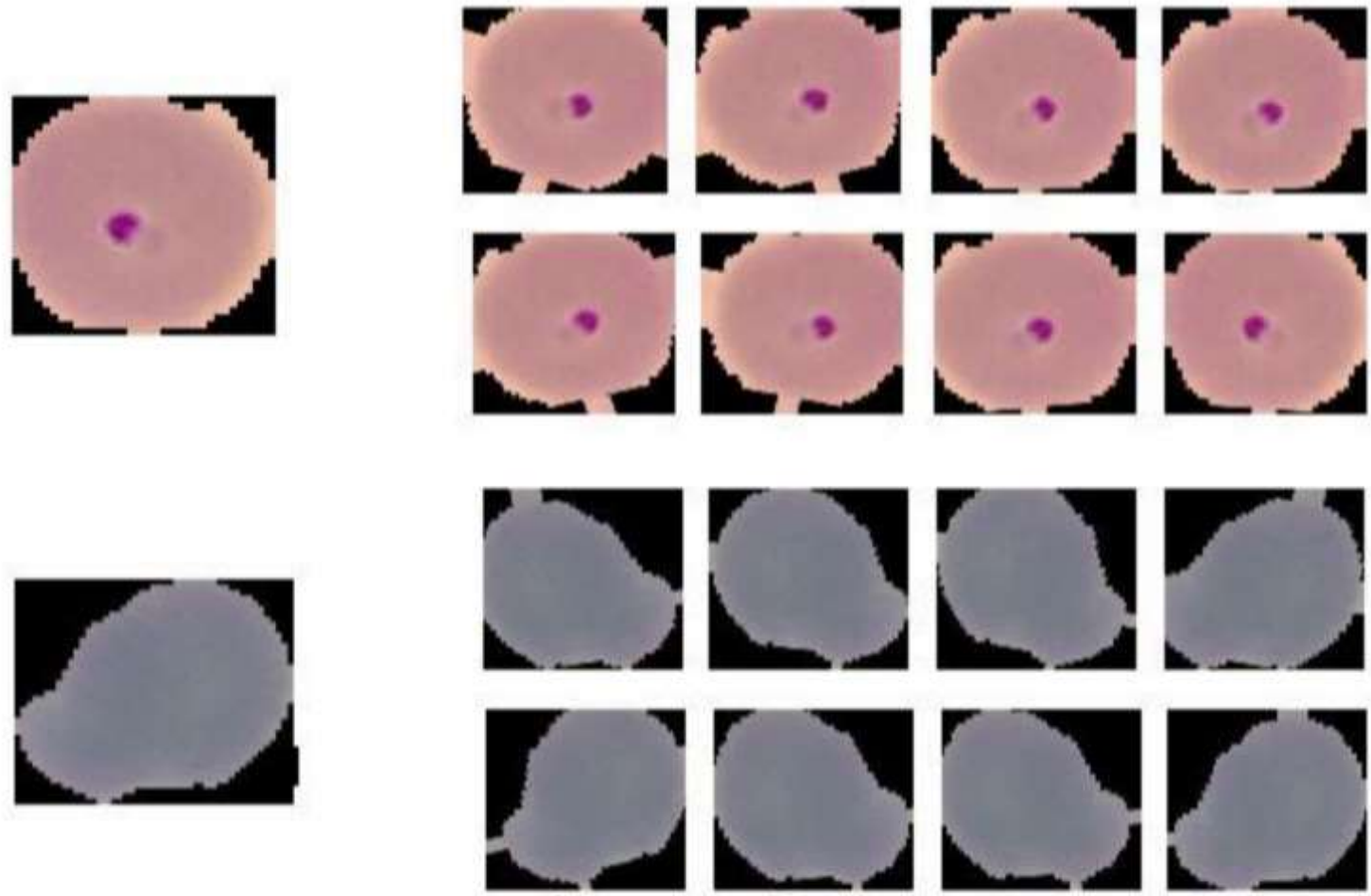

(a)

(b)

Fig. 1: Classification of infected and uninfected images

\section{Feature Extraction}

Feature extraction is the following stage in the process where the portioned picture from the past advance is given as an info. This procedure lessens the measure of information to be stacked by removing just the highlights those are required. The highlights incorporate difference, relationship, homogeneity, energy, shading histogram, shading minutes, etc. As the quantity of highlights increment the degree of exactness additionally increments.

The various highlights utilized in the writing to depict the presence of red platelets, contaminated and uninfected, in slim smears. Clearly, on the grounds that parasites have been stained, shading highlights are generally regular and undoubtedly utilized by numerous articles. Likewise, a few surface and morphologic highlights have been utilized to depict within red platelets. The thought is that in the event of contaminated cells, these highlights can get the common appearance of ring structures with noticeable cytoplasm and other exceptional parasite attributes. As a rule, the majority of the highlights utilized are attempted and confided in highlights that have effectively been applied in other, regularly nonmedical, application areas. For instance, Haralick's surface highlights, nearby paired examples, co-occurence lattices, histogram of angles, and numerous others have been effectively utilized across a wide scope of uses. This likewise incorporates morphologic shape highlights and minutes.

\section{Contrast}

It is the distinction in the luminance or shading which makes the pictures discernable. It is gotten by the adjustment of splendor of the picture or article starting with one pixel then onto the next.

\section{Correlation}

It is an activity which is utilized to remove data from pictures. The two key highlights: shiftinvariant and straight. Shift invariant plays out a similar activity at each point on the picture and straight replaces each pixel of the picture with that of its neighbors .

\section{Homogeneity}

A picture that is uniform in piece or character alludes to homogeneity in pictures. It is determined as follows homogeneity = graycoprops $($ graycomatrix $(i m g)$, 'Homogeneity') 


\section{Energy}

Energy decides the force of the pixels. The most extreme energy of a pixel is acquired by

maxGrayLevel $=\max ($ grayImage $(:))$ Color Histograms

It represents the distribution of colors in an image. For digital images, it represents the number of pixels that have colors in each of fixed ranges of colors .

\section{Classification}

The grouping strategy is finished by utilizing multi-class support vector machines (msvm). It is a procedure where in cases are grouped into at least one classes dependent on their comparative properties. It orders utilizing one-against-one methodology. SVM procedure is likewise executed and utilized for the grouping of the prepared pictures in order to look at and see the outcomes acquired through both SVM and the MSVM classifiers

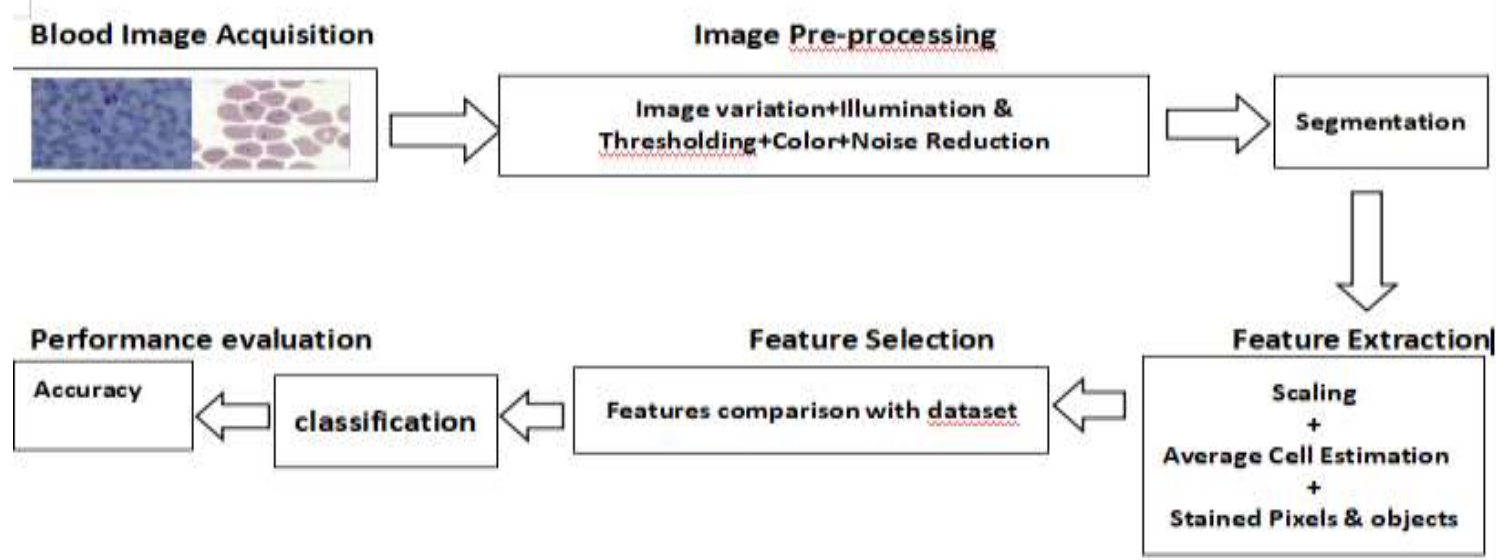

Fig2. Flowchart to identification of infected and uninfected images

\section{RESULTS}

To foster a proficient and exceptionally precise model for the recognition of the intestinal sickness parasite from fragmented cell pictures, a progression of tests including both AI and profound learning methods were performed. 

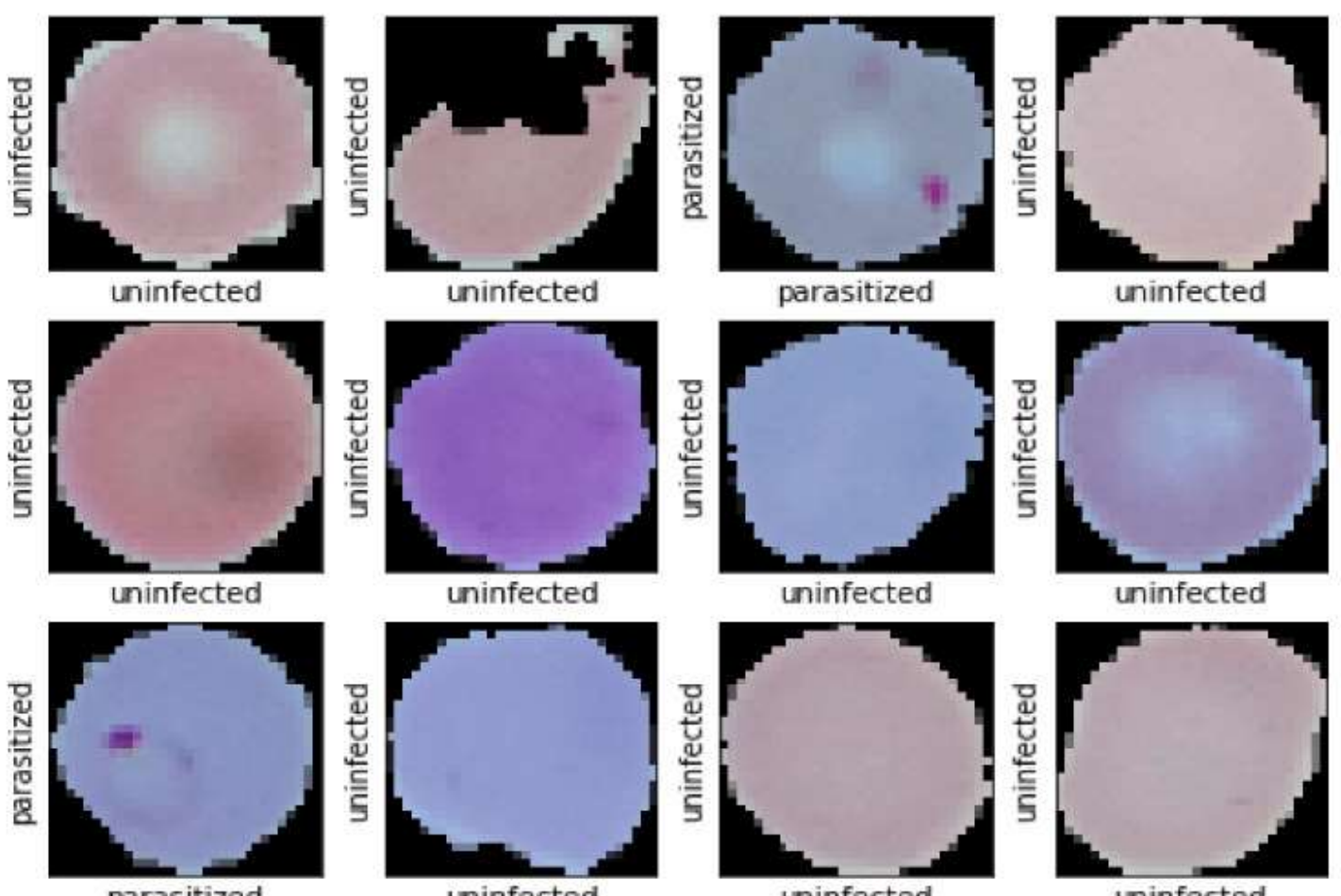

Fig. 3: Identification Of Infected Malarial Parasite

\section{CONCLUSION}

The reason for this paper is to foster a precise, fast and moderate framework for the recognizable proof of parasites of malaria in slender pictures of blood utilizing ImageProcessing. Here, minute bloodImages of typical, malaria tainted are procured, which is preprocessed and segmented.Certain highlights are separated from the sectioned picture inorder to decrease the measure of information to be held for investigation. From there on svm and msvm classifiers are utilized to identify whether the tiny picture of the platelets is intestinal sickness contaminated or not as the end-product.

\section{ACKNOWLEDGEMENT}

This research was supported by the S J Vivekanandan Assistant Professor Dhanalakshmi college of Engineering is acknowledged for supporting a research assistant to conduct the research. 


\section{REFERENCES}

[1] Deepali A. Ghate, Prof. Chaya Jadhav, Automatic Detection of Malaria Parasite from Blood Images International Journal of Computer Science and Security, vol.5, issue.3, pp.310-315, May 2017.

[2] Mahdieh Poostchi, Kamolrat Silamut, Richard Maude, Stefan Jaeger, George Thoma, Image analysis and machine learning for detecting malaria, Translational Research (2018), TRSL 1210 S1931-5244(17)30333-X.

[3] Malaria Parasite Detection Using Different Machine Learning Classifier -Adedeji Olugboja, Zenghui Wang - Machine College of Science, Engineering and Technology, University of South Africa

[4] Bias, S.D., Reni, S.K., Kale, I.: A novel fuzzy logic inspired edge detection technique for analysis of malaria infected microscopic thin blood images. In: 2017 IEEE Life Sciences Conference (LSC), Sydney, NSW, 2017, pp. 262-265.

[5] Mehanian, C., et al.: Computer-automated malaria diagnosis and quantitation using convolutional neural networks. In: 2017 IEEE International Conference on Computer Vision Workshops (ICCVW), Venice, 2017, pp. 116-125. 